\title{
In Silico Study on Binding Specificity of Gonadotropins and Their Receptors: Design of a Novel and Selective Peptidomimetic for Human Follicle Stimulating Hormone Receptor
}

\author{
Archana Sonawani ${ }^{9}$, Sarfaraj Niazi ${ }^{9}$, Susan Idicula-Thomas* \\ Biomedical Informatics Center of Indian Council of Medical Research, National Institute for Research in Reproductive Health, Mumbai, India
}

\begin{abstract}
Gonadotropins bind to specific receptors in spite of sharing a high level of sequence and structural similarity. This specific binding is crucial for maintaining the reproductive health of an organism. In this study, residues that dictate the receptor binding specificity of the gonadotropins (FSH and LH) have been identified using combination of in silico methods. Docking studies (ZDOCK), based on the systematic replacement of these residues, confirmed its importance in receptor binding. An interesting observation is that the relative positioning of the residues conferring binding specificity varied for the gonadotropin-receptor complexes. This spatial difference of the key residues could be exploited for design of specific modulators. Based on the identified residues, we have rationally designed a peptidomimetic (FSHP) that displays good binding affinity and specificity for hFSHR. FSHP was developed by screening 3.9 million compounds using pharmacophoreshape similarity followed by fragment-based approach. It was observed that FSHP and hFSHâ can share the same receptor binding site thereby mimicking the native hFSHR-FSH interactions. FSHP also displayed higher binding affinity to hFSHR as compared to two reported hFSHR antagonists. MD simulation studies on hFSHR-FSHP complex revealed that FSHP is conformationally rigid and the intermolecular interactions are maintained during the course of simulation.
\end{abstract}

Citation: Sonawani A, Niazi S, Idicula-Thomas S (2013) In Silico Study on Binding Specificity of Gonadotropins and Their Receptors: Design of a Novel and Selective Peptidomimetic for Human Follicle Stimulating Hormone Receptor. PLoS ONE 8(5): e64475. doi:10.1371/journal.pone.0064475

Editor: Paul Taylor, University of Edinburgh, United Kingdom

Received January 29, 2013; Accepted April 15, 2013; Published May 20, 2013

Copyright: (C) 2013 Sonawani et al. This is an open-access article distributed under the terms of the Creative Commons Attribution License, which permits unrestricted use, distribution, and reproduction in any medium, provided the original author and source are credited.

Funding: This work (NIRRH/M/77/12) has been supported by grant from Department of Biotechnology; Government of India (BT/PR12904/BID/07/301/2009). The funders had no role in study design, data collection and analysis, decision to publish, or preparation of the manuscript.

Competing Interests: The authors have declared that no competing interests exist.

*E-mail: thomass@nirrh.res.in

9 These authors contributed equally to this work.

\section{Introduction}

G-protein coupled receptors (GPCRs) form the largest family of integral membrane proteins. These proteins are evolutionarily conserved and share a common seven $\alpha$-helical transmembrane domain in their structures [1-3]. They are activated by a large number of diverse ligands such as small compounds, peptides or large proteins. The activated cell surface receptors transmit signals that induce a cellular response to the environment [4]. GPCRs are involved in many diseases and therefore are important drug targets [5-8].

The gonadotropin receptors, which include follicle-stimulating hormone receptor (FSHR) and lutropin/choriogonadotropin receptor (LH/CGR), belong to the class A group of GPCRs. The transmembrane domains (TMD) display high sequence identity $(\approx 70 \%)$ whereas the ectodomains are less similar $(\approx 40 \%)$. The gonadotropins viz., FSH, LH and CG are heterodimeric glycoprotein hormones. They have a common $\alpha$ subunit and a hormone specific $\beta$-subunit $[9,10]$. They are highly specific towards their cognate receptors, in spite of sharing high level of structural similarity. While FSH binds specifically to FSHR, the high sequence similarity $(\approx 80 \%)$ in the $\beta$-subunits of $\mathrm{LH}$ and $\mathrm{CG}$ enables them to share a common receptor (LH/
GGR). The binding of gonadotropins to their receptors initiates a signalling cascade which eventually brings about maturation of ovarian follicles in females and spermatogenesis in males [11]. These interactions are therefore crucial for regulating reproduction and gonadal development.

The extracellular domain (ECD) of the receptors and the $\beta$ subunit of the hormones has been experimentally shown to govern binding specificity $[12,13]$. In the absence of the crystal structure of the complete hormone-receptor complex, several experimental studies using different approaches have been undertaken to delineate the residues important for binding specificity. In case of receptors, chimera studies have shown that $\beta$-strands 3 and 6 of human LHR (hLHR) are important for dictating the binding selectivity [14]. Alanine scanning and mutation studies for all the residues of these two $\beta$-strands revealed that residues $104 \mathrm{~N}$ of $\beta$ strand 3 and $179 \mathrm{G}$ of $\beta$-strand 6 contribute to the selectivity of binding of hLHR to hLH/CG. Thus, introduction of residue $104 \mathrm{~N}$ in hFSHR promotes hLH/CG binding to hFSHR whereas $179 \mathrm{~K}$ of hFSHR prevents this binding [15]. Introduction of $\beta$ strand 1 of hFSHR into hLHR led to its binding to hFSH and introduction of a combination of $\beta$-strand 1 with few other $\beta$ strands of hFSHR into hLHR increased its binding affinity to hFSH. These observations suggested that residues belonging to 
multiple strands of hFSHR contribute towards binding selectivity [16].

Chimeric hCG/FSH $\beta$-subunits were constructed and analysed for their ability to interact with hLHR and hFSHR as well as hormone specific monoclonal antibodies. Substitution of 33-52 residues of hFSH with 39-58 residues of hCG showed no effect on receptor binding. However, substitution of 94-145 of hCG $\beta$ with 88-108 of hFSH $\beta$ resulted in a hormone analogue identical to hFSH in its ability to bind to hFSHR [17]. To further delineate the residues of this region that dictate binding specificity, 88DSDS91 and 95TVRGLG100 regions of hFSH were replaced by hLH residues 94RRST97 and 101GGPKDH106 respectively. The first substitution did not affect hFSHR binding but conferred hLHR binding to the chimera. The second substitution caused loss of binding to both hFSHR and hLHR. The study reveals that hFSH residues 95TVRGLG100 are crucial for FSH binding specificity whereas hLH residues 94RRST97 are involved in conferring hLHR binding specificity [18]. The results suggest that the regions/residues that contribute to binding specificity differ for the gonadotropins and several residues contribute to binding specificity. In such cases, where the binding surface is large, the knowledge of important residues involved in protein-protein interaction is exploited to design peptidomimetic modulators [19-21].

Various strategies have been used to design peptidomimetics, such as, insertion of unnatural amino acids, introduction of conformational constraints, isostearic replacement of peptide bonds [22], inversion of amino acid sequences and $\alpha$-carbon chirality [23], screening and identification of suitable scaffolds based on shape and pharmacophore based similarity, followed by fragment-based approach [24]. Peptidomimetics have been successfully designed for various therapeutically important GPCR targets such as neuropeptide PLG analogs for modulating dopamine receptor [25], Pasireotide (SOM230) mimic somatostatin [26], Aba-Gly scaffold-based peptidomimetic for $\mu$-opioid receptor [27], cyclic $\alpha-\mathrm{MSH}$ analogs for melanocortin-4 receptor [28] and orally active GnRH antagonist AEZS-115 [29]. Efforts to design successful FSHR peptidomimetic modulators are still ongoing [30].

Since FSHR-FSH interaction is crucial for gonadotropin action, molecules that can block or mimic this interaction can serve as fertility regulating agents. This study aims to delineate the residues of gonadotropins and their receptors that influence binding specificity based on the available sequence and structural information and leverage this information for the rational design of FSH peptidomimetic. This is the first comprehensive structurebased study aimed at designing FSH peptidomimetics using the knowledge of hFSHR-FSH $\beta$ binding specific residues. The designed FSH peptidomimetic was found to have good binding affinity, stability and selectivity to hFSHR as assessed by docking followed by MD simulation studies. The information gained by this study could aid in the rational design of fertility regulating agents.

\section{Methods}

\section{Dataset creation}

Sequences of gonadotropins (FSH and $\mathrm{LH}$ ) and their receptors (FSHR and LHR) found in various organisms were retrieved from Protein database of NCBI (Table 1). The sequence information was restricted to $\beta$-subunit of hormones and the ECD region of receptors.
Table 1. Sequence datasets of gonadotropins and their receptors.

\begin{tabular}{ll}
\hline Gonadotropins/Receptors & No. of sequences \\
\hline FSH & 21 \\
LH & 21 \\
FSHR & 16 \\
LHR & 9 \\
\hline doi:10.1371/journal.pone.0064475.t001
\end{tabular}

\section{Structural analysis}

The crystal structure of hFSHR-FSH was retrieved from Protein Data Bank (PDB ID: 1XWD), while the modeled, energy minimized structure of hLHR-LH was retrieved from Glycoprotein-hormone Receptors Information System database (GRIS) [31]. The modeled hLHR-LH complex structure was validated using Structural Analysis and Validation Server (SAVES, http:// nihserver.mbi.ucla.edu/SAVES/). Recently, the entire ectodomain of hFSHR complexed with FSH has been elucidated (PDB ID: 4AY9). It is to be noted that the hormone-receptor interface of 4AY9 and 1XWD are identical. The $\beta$-subunit of hormones and ECD region of receptors were considered for analyses. The interactions that stabilize the hormone-receptor complex were identified using Protein Interactions Calculator (PIC) [32]. The hydrogen bonds and salt bridges were computed using HBPLUS [33] and Evaluating the Salt BRIdges in Proteins (ESBRI) [34] softwares respectively. The default parameters were used for identification of the interactions. The first and second shell residues of the interface region were considered as crucial for the hormone-receptor binding.

\section{Sequence analysis}

The residues identified to be critical in stabilizing the hormonereceptor complex based on the structural analyses were evaluated for evolutionary conservation and uniqueness to the gonadotropin and the receptor family. Using in-house codes, the sequence information of gonadotropins and receptors was further simplified and reduced based on the physico-chemical properties of amino acids. The amino acids were grouped into neutral (N, Q S, T), acidic $(\mathrm{D}, \mathrm{E})$, basic $(\mathrm{K}, \mathrm{R})$, aromatic $(\mathrm{F}, \mathrm{Y}, \mathrm{W})$ and aliphatic $(\mathrm{V}, \mathrm{A}$, L, I, M) groups. C, P, H and $\mathrm{G}$ amino acids were considered independently.

Table 2. Mutants created for gonadotropins and receptors.

\begin{tabular}{llll}
\hline hFSHRm & hLHRm & hFSHm & hLHm \\
\hline E50R & A54V & P42V & R89S \\
R52S & Y55L & S89R & P97R \\
V54A & L101R & D90S & K98G \\
L55Y & Q103E & T95G & D105S \\
R101L & C128S & V96G & - \\
E103Q & Y178N & R97P & - \\
K104N & E199N & Y103T & - \\
K179G & - & - & - \\
V221K & - & - & - \\
\hline doi:10.1371/journal.pone.0064475.t002 &
\end{tabular}




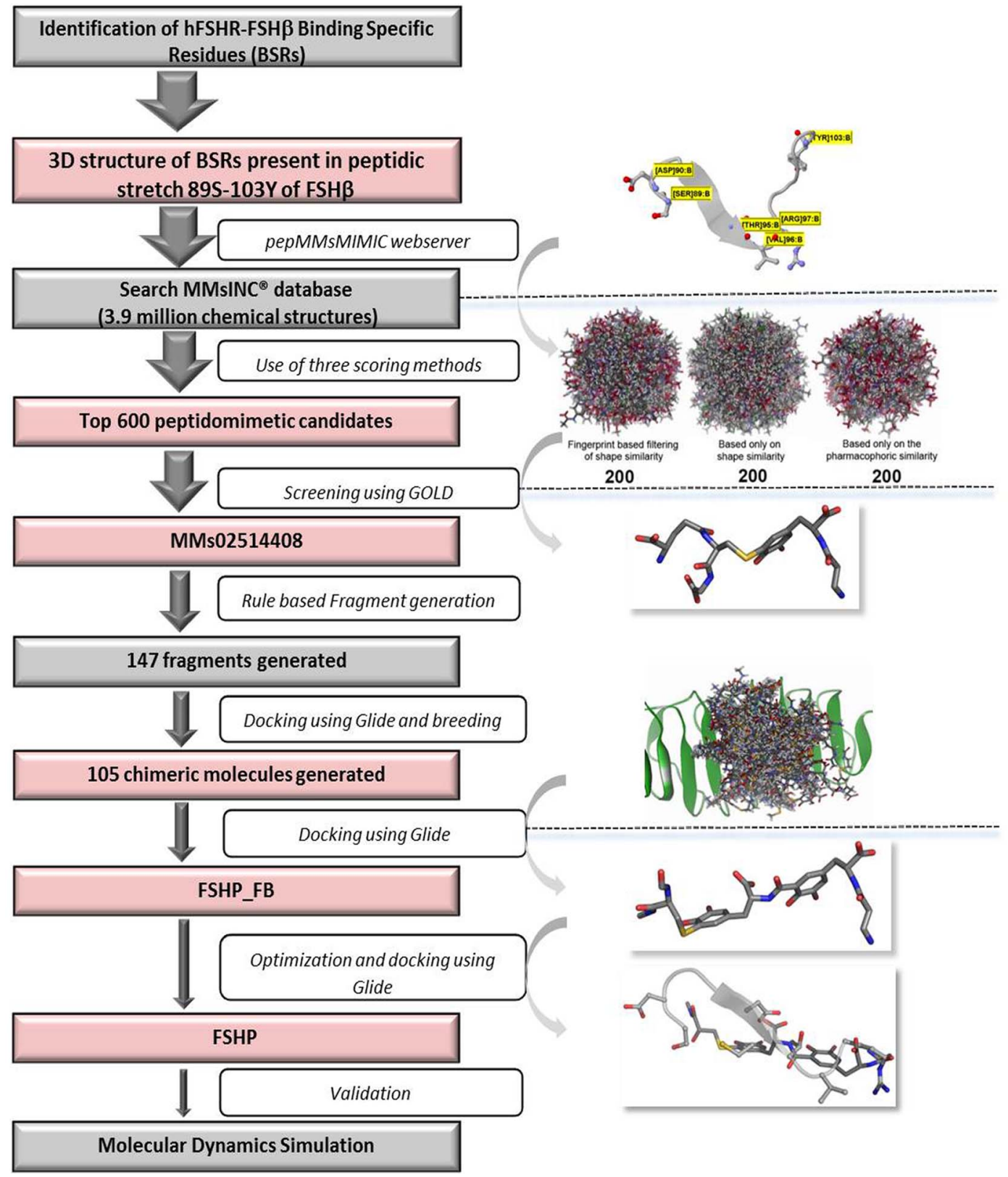

Figure 1. Flowchart describing the rational design of FSH peptidomimetic (FSHP). The structures represent information provided in pink boxes.

doi:10.1371/journal.pone.0064475.g001

BioEdit [35] version 5.0.9 was used to generate the pairwise and multiple sequence alignments (MSA). These alignments were validated by their corresponding structural alignments obtained using DSSP program [36].

\section{Generation of mutant structures}

The in silico mutants were generated using Build Mutant protocol of Accelrys Discovery studio 2.0 (Acc. DS 2.0) and further energy minimized using 200 steps of steepest descent algorithm. The mutant hFSH (hFSHm) was generated by substituting all the residues of hFSH, identified to be important for hFSHR-FSH binding, with the corresponding residues of hLH. Mutant hFSHR (hFSHRm) was generated by substituting all the residues of hFSHR, identified to be important for hFSHR-FSH binding, with the corresponding residues of hLHR. Likewise, mutant hLH (hLHm) and mutant hLHR (hLHRm) were also generated (Table 2). The corresponding residue positions of the gonadotropins and their receptors were identified based on their sequence and structural alignments.

\section{Molecular docking}

Protein-protein docking. The structural coordinates of hFSHR-FSH and hLHR-LH were used for docking. The water molecules and hetero atoms were removed and the molecules were subjected to energy minimization using 200 steps of steepest descent algorithm. The energy minimised structures were docked using ZDOCK algorithm (Acc. DS 2.0). 
Table 3. List of interactions that stabilize the gonadotropin-receptor complexes.

\begin{tabular}{|c|c|c|c|}
\hline hFSHR Second* - First shell & Interaction & hFSHR First shell - hFSH ${ }^{\infty}$ & Interaction \\
\hline $52 R-50 \mathrm{E}$ & Electrostatic & $50 E-\mathbf{9 7 R}$ & Electrostatic \\
\hline $54 V-55 \mathrm{~L}$ & Hydrophobic & $55 L-\mathbf{1 0 3 Y}$ & Hydrophobic \\
\hline $103 E-101 R$ & Salt bridge & $101 R-96 V$ & Hydrophobic \\
\hline $103 E-104 \mathrm{~K}$ & Electrostatic & $104 \mathrm{~K}-95 \mathrm{~T}$ & Hydrogen bond \\
\hline- & - & $179 \mathrm{~K}-\mathbf{8 9 S}$ & Hydrogen bond \\
\hline - & - & 179K - 90D & Salt bridge \\
\hline $222 \mathrm{l}-221 \mathrm{~V}$ & Hydrophobic & $221 V-\mathbf{4 2 P}$ & Hydrophobic \\
\hline hLHR Second*- First shell & Interaction & hLHR First shell - hLH ${ }^{\infty}$ & Interaction \\
\hline $54 A-55 L$ & Hydrophobic & $55 L-105 D$ & Hydrogen bond \\
\hline - & - & 101L - 97P & Hydrophobic \\
\hline $128 C-103 Q$ & Hydrogen bond & $103 Q-\mathbf{9 8 K}$ & Hydrogen bond \\
\hline - & - & $178 Y-89 R$ & Cation- $\pi$ \\
\hline- & - & 199E - 89R & Electrostatic \\
\hline
\end{tabular}

*The second shell residues of the receptors are italicised. ${ }^{\infty}$ Interacting gonadotropin residues are represented in bold.

doi:10.1371/journal.pone.0064475.t003

For each of the docking simulations, 50 poses belonging to 10 clusters were generated. Clusters with maximum number of poses and/or whose cluster centre has $\mathrm{C} \alpha$ root mean square deviation (RMSD) within $10 \AA$ and RDOCK energy less than $10 \mathrm{Kcal} / \mathrm{mol}$ were shortlisted. The structural stability (based on the RDOCK energy) and presence of native-like interactions were used to identify the best pose from each of the shortlisted clusters, which were further used for structural analyses.

Peptidomimetic screening. GOLD [37,38] v5.0 was used for initial screening of peptidomimetics retrieved from pepMMsMIMIC server [39]. The hFSHR (ECD) was prepared by removing hetero atoms and water molecules. Polar and non-polar hydrogen atoms, including those necessary to define the correct ionization and tautomeric states of residues were added. The binding site was defined by selecting $\mathrm{O} \varepsilon 1$ of $103 \mathrm{E}$ as the centroid atom and the binding site radius was set to $15 \AA$ so as to encompass all the binding specific residues (BSRs). Default settings were used for docking the peptidomimetics. The top-ranked solutions obtained from each genetic algorithm run were then screened for identifying the peptidomimetic that shared maximum number of interactions with BSRs.

Protein peptidomimetic docking. Protein peptidomimetic docking simulations were performed using Glide Extra-Precision (XP) mode [40,41]. The structures of the receptors and the peptidomimetics were prepared using Protein Preparation Wizard and LigPrep [42] v2.5 applications of Maestro [43] v9.2 respectively, using default settings. Receptor grids were generated for hFSHR using coordinates $\mathrm{X}=-1.970 ; \mathrm{Y}=-23.420$; $\mathrm{Z}=30.930$ and hLHR using coordinates $\mathrm{X}=-1.106$; $\mathrm{Y}=-22.327 ; \mathrm{Z}=31.732$. The structures were energy minimised using OPLS2005 forcefield. Poses were selected based on GlideScore, Model energy (Emodel) score and interactions with BSRs.

\section{Rational design of FSH Peptidomimetic}

Pharmacophore-shape similarity based virtual screening of MMsING ${ }^{\circledR}$ database. The $3 \mathrm{D}$ structure of FSH $\beta$ peptidic stretch $89 \mathrm{~S}-103 \mathrm{Y}$ that includes the BSRs except $42 \mathrm{P}$ (as it is topologically distant) was used as the template for pepMMsMIMIC, a web-based peptidomimetic compound virtual screening tool. The pharmacophore model, generated based on the BSRs, was screened against a library of 17 million conformers obtained from 3.9 million commercially available chemical structures present in MMsINC $^{\circledR}$ database [44]. The pepMMsMIMIC webserver employs five types of scoring methods to optimize the selection of the peptide mimetics, of which we have used viz., 1) fingerprint based filtering of shape similarity 2) based only on shape similarity and 3) based only on the pharmacophoric similarity. The 600 (top 200 per scoring method) peptidomimetics retrieved from pepMMsMIMIC were further screened by docking with hFSHR using GOLD. The best peptidomimetic identified by GOLD (MMs02514408) was further docked using Glide to assess the reproducibility of results.

Generation of novel peptidomimetics using fragmentbased approach and optimization. The fragment-based approach for generation of novel peptidomimetics was carried out using the Fragmentor script, BREED and Glide tools available in Schrödinger.

MMs02514408 was fragmented using the Fragmentor script [45] and the fragments were docked with hFSHR using Glide. The BREED algorithm was used to hybridise the docked fragments and develop novel, chimeric molecules [46]. These chimeric molecules were then docked with hFSHR. The docked poses were analyzed with respect to their binding orientation, affinity and intermolecular interactions to identify the best peptidomimetic. The identified peptidomimetic was further modified by substituting solvent accessible groups by various functionalities to reduce the molecular weight and increase the binding affinity for hFSHR.

The twelve peptidomimetic derivatives thus generated, were then docked to hFSHR using Glide. The docked complexes were energy minimized using CHARMM forcefield with 200 cycles of conjugate gradient algorithm (DS 2.0). Generalized-Born was selected as an implicit solvent model while other parameters were kept default. The non-bonded interaction energies were specifically calculated between the BSRs and the docked peptidomimetics. Thus, the FSH peptidomimetic was designed and optimised using pepMMsMIMIC and fragment-based approach as shown in flowchart (Figure 1). 

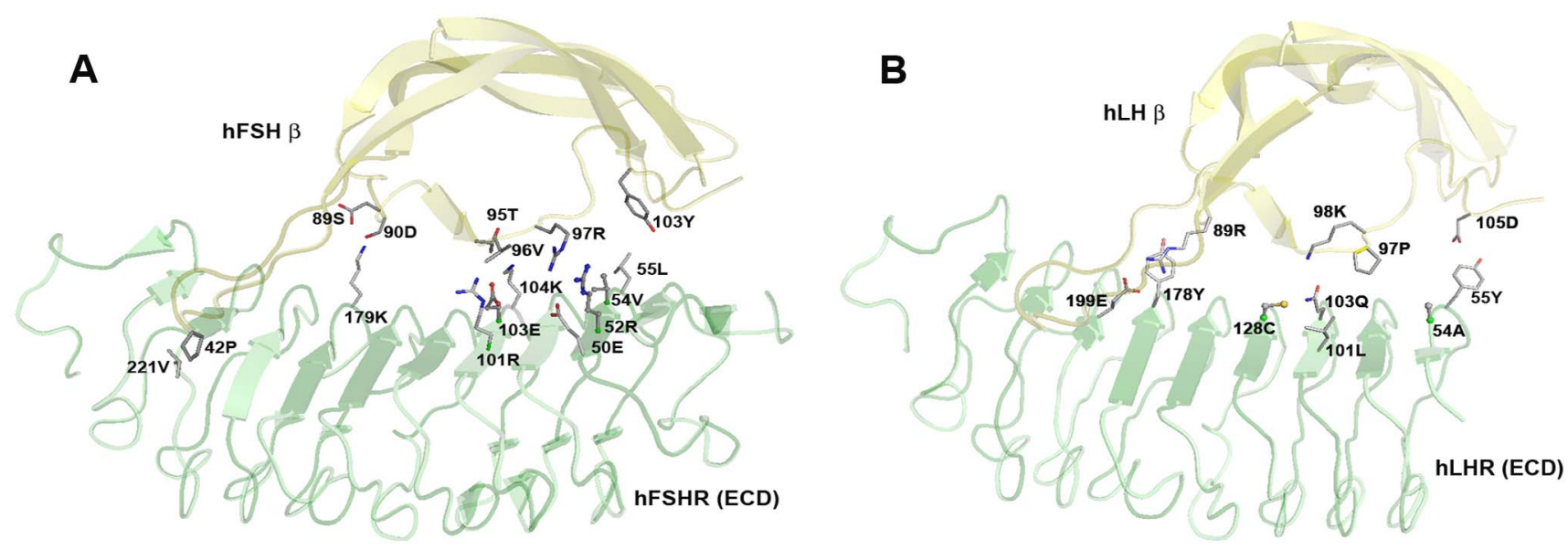

Figure 2. Binding specificity residues identified for (A) hFSHR-FSH complex and (B) hLHR-LH complex. The first shell residues are represented by sticks and the second shell residues are represented by ball and stick. The gonadotropins are colored yellow and the receptors are colored green.

doi:10.1371/journal.pone.0064475.g002

The structures were rendered using DS 2.0 and PyMol version 1.2 (http://www.pymol.org). Chemical structures were drawn using ChemDraw Ultra v8.0 [47].

\section{Molecular Dynamic Simulations}

Molecular Dynamic (MD) simulations were performed for the docked complex of hFSHR-FSHP using GROMACS version 4.5.4 [48] with the implementation of the CHARMM forcefield [49]. The topology file for FSHP was generated by SwissParam using CHARMM all atoms forcefield [50]. The van der Waal interactions were calculated with a distance cut-off of $0.9 \mathrm{~nm}$. Particle Mesh Ewald (PME) summation was applied for long range electrostatics with $1 \mathrm{~nm}$ cut-off for columbic interactions [51]. Counterions were added to neutralize the system. The system was solvated using SPG water model [52] and simulated in an octahedron box with periodic boundary conditions. The structures were first energy minimized using steepest descent algorithm with a tolerance of $1000 \mathrm{KJmol}^{-1} \mathrm{~nm}^{-1}$. The system was equilibrated by applying position restraints on the complex and performing simulations using canonical NVT ensemble followed by NPT. Both the simulations were run for $100 \mathrm{ps}$ each at a temperature of $300 \mathrm{~K}$. Temperature coupling was performed using velocity

Table 4. Binding specific residues identified for gonadotropin-receptor complexes.

\begin{tabular}{llll}
\hline hFSHR & hFSH & hLHR & hLH \\
\hline $50 \mathrm{E}$ & $42 \mathrm{P}$ & $55 \mathrm{Y}$ & $89 \mathrm{R}$ \\
$55 \mathrm{~L}$ & $89 \mathrm{~S}$ & $101 \mathrm{~L}$ & $97 \mathrm{P}$ \\
$101 \mathrm{R}$ & $90 \mathrm{D}$ & $103 \mathrm{Q}$ & $98 \mathrm{~K}$ \\
$104 \mathrm{~K}$ & $95 \mathrm{~T}$ & $178 \mathrm{Y}$ & $105 \mathrm{D}$ \\
$179 \mathrm{~K}$ & $96 \mathrm{~V}$ & $199 \mathrm{E}$ & - \\
$221 \mathrm{~V}$ & $97 \mathrm{R}$ & $54 \mathrm{~A}$ & - \\
$52 R$ & $103 \mathrm{Y}$ & $128 \mathrm{C}$ & - \\
$54 \mathrm{~V}$ & - & - & - \\
$103 E$ & - & - & - \\
\hline The second-shell residues are italicized. & \\
doi:10.1371/journal.pone.0064475.t004 &
\end{tabular}

rescaling [53] with a coupling constant of $0.1 \mathrm{ps}$ and the initial velocities were generated according to Maxwell distribution. Temperature-pressure coupling was performed using extendedensemble Parrinello-Rahman algorithm [54] with a coupling constant of 2 ps. The equilibrated system was then subjected to 5 ns of production run. A time step integration of $2 \mathrm{fs}$ was used. The trajectories were saved every 500 steps and analysed using GROMACS analysis tools and XMGRACE-5.1.22 program (http://plasma-gate.weizmann.ac.il/Grace/).

\section{Results and Discussion}

\section{Identification of residues important for binding specificity}

The crystal structure of hFSHR-FSH complex is available in PDB (PDB ID: 1XWD, 4AY9) [55,56] and the theoretical structure of hLHR-LH is available in the GRIS database. The quality of the hLHR-LH model was assessed using SAVES and it was found to be acceptable for further studies (See Table S1). The crystal structure of hFSHR (ECD)-FSH $\beta$ and the modeled structure of hLHR (ECD)-LH $\beta$ were analyzed to identify residues that contribute to the binding and stability of the hormonereceptor interactions. Since, the receptor residues that do not directly interact with the hormone i.e. the second shell residues could also influence hormone binding [57], the first and second shell residues in the hormone-receptor interface region were identified and the nature of their interactions was studied using PIC (Table 3).

The sequence information of gonadotropins and their receptors from various organisms were analysed to understand the degree of

Table 5. RDOCK energy of the docked complexes.

\begin{tabular}{llll}
\hline & & & \\
\hline & $\begin{array}{l}\text { Energy (Kcal/ } \\
\text { mol) }\end{array}$ & Docked complex & $\begin{array}{l}\text { Energy (Kcal/ } \\
\text { mol) }\end{array}$ \\
\hline hFSHR-hFSH & -34.16 & hLHR-hLH & -30.47 \\
hFSHR-hFSHm & 14.3 & hLHR-hLHm & -12.0 \\
hFSHRm-hFSH & 23.4 & hLHRm-hLH & 10.3 \\
\hline doi:10.1371/journal.pone.0064475.t005 & &
\end{tabular}



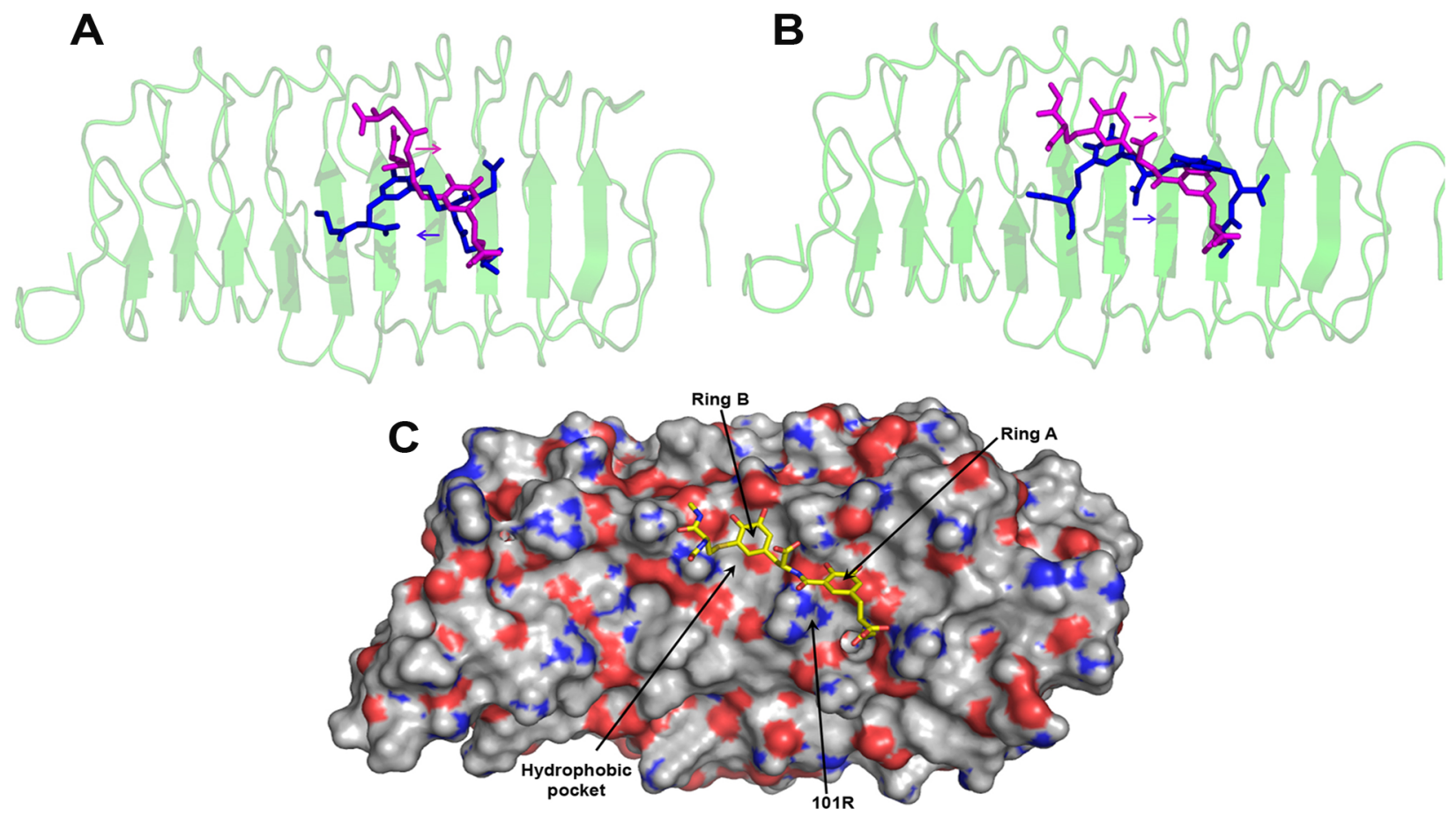

Figure 3. Binding modes of MMs02514408 and FSHP_FB predicted by GOLD and Glide. (A) Opposite binding modes predicted for MMs02514408 with hFSHR (B) Similar binding modes predicted for FSHP_FB with hFSHR. The GOLD and Glide docked poses are shown as blue and pink sticks respectively. hFSHR is depicted in green cartoon representation. (C) Figure illustrating the importance of two planar hydrophobic groups (phenyl rings A and B) present in FSHP_FB (yellow sticks) for hFSHR binding (Molecular surface representation). Ring A forms cation- $\pi$ interaction with $101 \mathrm{R}$ while ring $\mathrm{B}$ is embedded in the hydrophobic pocket of the binding site.

doi:10.1371/journal.pone.0064475.g003

conservation for each of the residues identified from structural analysis. The corresponding residue positions of gonadotropins and their receptors were obtained from the sequence and structure-based alignments. The reduced alphabet representation of the sequences, based on the physicochemical properties of amino acids, helped to delineate residues that were strictly conserved and unique in the homologous sequences of a given family of gonadotropins and their receptors. For e.g. the basic residue $(\mathrm{K} / \mathrm{R})$ at position 97 is conserved in $\mathrm{FSH}$ while at the corresponding position, $\mathrm{P}$ is conserved in LH (Figure S1). As

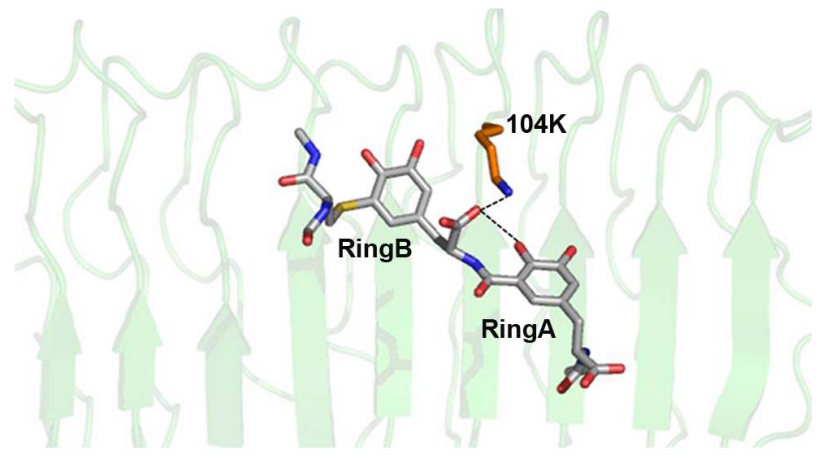

Figure 4. Inter and intra molecular interactions provide rigidity to the spacer connecting rings $\mathbf{A}$ and $\mathbf{B}$. Anionic carboxyl oxygen atom of FSHP_FB (gray sticks) is involved in intramolecular hydrogen bond formation with hydroxyl group present at the ortho-position of Ring A and also forms salt bridge with 104K (orange stick) of hFSHR (green cartoon). The interactions are shown in black dotted lines. doi:10.1371/journal.pone.0064475.g004 expected, the conservation of the physicochemical nature of residues of the gonadotropins is further reflected in the nature of the interacting residues of its cognate receptor. $97 \mathrm{R}$ of $\mathrm{hFSH} \beta$ is involved in electrostatic interaction with $50 \mathrm{E}$ of hFSHR. $50 \mathrm{E}$ is stabilized by intra-molecular electrostatic interactions with $52 \mathrm{R}$ (second-shell residue) of hFSHR. Instead of an acidic residue (D/ E) at position 50, which is conserved in FSHR, a basic residue (K/ $\mathrm{R})$ is conserved in LHR at the corresponding position. Similarly, other residues that are crucial for binding specificity were delineated based on structural analysis and sequence conservation (Figure 2 and Table 4).

\section{Validation of the BSRs by docking studies}

Mutants for the gonadotropins and receptors were created based on the residues identified to be critical for binding specificity from sequence and structural analysis. The mutant and wild-type structures of the gonadotropins and their receptors were docked and the energies of the complexes compared to understand the contribution of the identified residues to binding stability. The docking algorithm was initially validated by re-docking the wildtype structures, which correctly reproduced the X-ray crystal structure of hFSHR-FSH (1XWD) and the modeled structure of hLHR-LH with RMSD of $0.98 \AA$ and $1.3 \AA$ respectively (Figure $\mathrm{S} 2)$.

ZDOCK generated eight to ten clusters containing several docked poses for each of the 6 complexes and ranked them according to their ZDock score. Subsequent to RDOCK refinement, clusters were short-listed for each of the docked complexes based on the selection/filtering criteria (see methods). The best pose within each of the selected cluster centers were then identified based on RDOCK energy and presence of native-like 
<smiles>CNC(=O)[C@H](NC=O)Sc1cc(C[C@H](NC(=O)c2cc(C[C@H](NC(=O)CCN)C(=O)O)cc(O)c2O)C(=O)O)cc(O)c1O</smiles><smiles>CNC(=O)C(CSc1cc(C[C@H](NC(=O)c2cc(C[C@H](NC(=O)CCN)C(=O)O)cc(O)c2O)C(=O)O)cc(O)c1O)C(F)(F)F</smiles><smiles>CNC(=O)[C@H](O)CSc1cc(C[C@H](NC(=O)c2cc(CC(NC(=O)CCN)C(=O)O)cc(O)c2O)C(=O)O)cc(O)c1O</smiles><smiles>CNC(=O)[C@H](Cc1cc(O)c(O)c(C(=O)N[C@@H](Cc2cc(O)c(O)c(SC[C@H](O)C(=O)NC)c2)C(=O)O)c1)NC(=O)CCN</smiles><smiles>CNC(=O)[C@H](CSc1cc(C[C@H](NC(=O)c2cc(C[C@H](NC(C)=O)C(=O)O)cc(O)c2O)C(=O)O)cc(O)c1O)NC(=O)CCN</smiles><smiles>CNC(=O)[C@H](O)CSc1cc(C[C@H](NC(=O)c2cc(C[C@@H](O)NC(=O)CCN)cc(O)c2O)C(=O)O)cc(O)c1O</smiles><smiles>CNC(=O)[C@H](N)CSc1cc(C[C@H](NC(=O)c2cc(C[C@H](NC(=O)CCN)C(=O)O)cc(O)c2O)C(=O)O)cc(O)c1O</smiles><smiles>CNC(=O)[C@H](O)CSc1cc(C[C@H](NC(=O)c2cc(CC(N)NC(=O)CCN)cc(O)c2O)C(=O)O)cc(O)c1O</smiles><smiles>CNC(=O)[C@H](CSc1cc(C[C@H](NC(=O)c2cc(CC(NC(=O)CCN)C(=O)O)cc(O)c2O)C(=O)O)cc(O)c1O)NC</smiles><smiles>CNC(=O)[C@H](O)CSc1cc(C[C@H](NC(=O)c2cc(CC(C)NC(=O)CCN)cc(O)c2O)C(=O)O)cc(O)c1O</smiles><smiles>CNC(=O)[C@H](C)CSc1cc(C[C@H](NC(=O)c2cc(C[C@H](NC(=O)CCN)C(=O)O)cc(O)c2O)C(=O)O)cc(O)c1O</smiles><smiles>CNC(=O)[C@H](O)CSc1cc(C[C@H](NC(=O)c2cc(C[C@@H](CN)NC(=O)CCN)cc(O)c2O)C(=O)O)cc(O)c1O</smiles><smiles>CNC(=O)C(C)CSc1cc(C[C@H](NC(=O)c2cc(CCNC(=O)CCN)cc(O)c2O)C(=O)O)cc(O)c1O</smiles>

Figure 5. Chemical structures of the peptidomimetics obtained by in silico structural modifications made at two stereo centers of FSHP_FB viz., $11 C \alpha_{1}$ and $53 C \alpha_{3}$. Substituent groups/atoms are highlighted in blue. FSHP_FB has four structural units viz., L-cysteine (orange), Dphenylalanine (green) and $\beta$-alanine (purple). doi:10.1371/journal.pone.0064475.g005

interactions. The docking studies revealed that the stability of the wild type complexes is remarkably higher than the mutants (Table 5). This observation validates the role of the identified residues in stabilizing the gonadotropin-receptor interactions.

\section{Observed cross-reactivity of gonadotropins could be explained by BSRs}

In spite of the interactions between gonadotropins and their receptors being highly selective within species, there have been reports of gonadotropin-receptor cross-reactivity observed amongst different species. For e.g. horse LH can bind to rat and all mammalian FSHRs [58,59], chicken LH binds with higher affinity to rat FSHR as compared to rat LHR [60] and hFSHR can be activated by porcine and bovine FSH at higher concentrations [61]. We sought an explanation to the first two cases of reported cross-reactivity of gonadotropins and receptors based on the residues that have been identified as important for binding specificity by our study.

We have identified that the presence of an aliphatic residue at 96 position of FSH is crucial for FSHR interaction (Figure 2). It is noteworthy that horse $\mathrm{LH}$ has $96 \mathrm{~V}$ (instead of $96 \mathrm{G}$ that is 
Table 6. Docking of peptidomimetics generated during optimisation of FSHP_FB.

\begin{tabular}{|c|c|c|c|c|}
\hline Peptidomimetic $^{\S}$ & Interacting BSRs of hFSHR & GlideScore (Kcal $/ \mathrm{mol})$ & EModel & Interaction Energy (Kcal/mol) \\
\hline FSHP_FB & 50E, 101R,103E 104K,179K & -7.83 & -67.44 & -180.86 \\
\hline FSHP & $52 \mathrm{R}, 101 \mathrm{R}, 103 \mathrm{E}, 104 \mathrm{~K}, 179 \mathrm{~K}$ & -7.98 & -75.40 & -180.90 \\
\hline FSHP_1 & 50E, 101R,103E, 104K & -7.83 & -67.44 & -184.06 \\
\hline FSHP_2 & $50 \mathrm{E}, 103 \mathrm{E}, 104 \mathrm{~K}$ & -8.62 & -82.54 & -110.81 \\
\hline FSHP_3 & $50 E, 101 R, 103 E$ & -6.83 & -73.42 & -108.22 \\
\hline FSHP_4 & $50 \mathrm{E}, 103 \mathrm{E}, 104 \mathrm{~K}$ & -8.88 & -77.22 & -163.14 \\
\hline FSHP_5 & $50 \mathrm{E}, 101 \mathrm{R}$ & -7.73 & -63.96 & -118.31 \\
\hline FSHP_6 & $50 \mathrm{E}, 103 \mathrm{E}, 104 \mathrm{~K}, 179 \mathrm{~K}$ & -6.82 & -79.06 & -145.94 \\
\hline FSHP_7 & $50 \mathrm{E}, 52 \mathrm{R}$ & -6.35 & -69.42 & -49.12 \\
\hline FSHP_8 & $50 \mathrm{E}, 104 \mathrm{~K}$ & -7.96 & -86.92 & -85.04 \\
\hline FSHP_9 & $50 \mathrm{E}, 103 \mathrm{E}$ & -5.88 & -67.53 & -104.00 \\
\hline FSHP_10 & $103 \mathrm{E}, 104 \mathrm{~K}$ & -7.71 & -75.32 & -81.62 \\
\hline FSHP_11 & 50E, 101R,103E & -6.70 & -76.83 & -52.16 \\
\hline
\end{tabular}

${ }^{\S}$ Chemical structures of the peptidomimetics have been shown in Figure 5 .

doi:10.1371/journal.pone.0064475.t006

conserved amongst LH) and probably for this reason, horse LH can bind to FSHR of other mammalian species (Figure S1). The observation that chicken LH binds with higher affinity to rat FSHR than rat LHR could be explained based on the following: a) the presence of a basic residue at 179 position of FSHR is important for FSH binding (Figure 2). Interestingly, chicken LHR also has a basic residue, lysine at position 179 instead of the conserved glycine seen at 179 position of LHR; b) Proline at 42 position of FSH is crucial for FSHR binding (Figure 2). Chicken LH has 42P instead of leucine which is conserved in LH.

Although these observations might give a plausible explanation to the observed cross-reactivity, further studies are to be carried

Table 7. Interactions between FSHP and hFSHR in the docked complex.

\begin{tabular}{|c|c|c|}
\hline hFSHR $^{\infty}$ & FSHP & Interaction \\
\hline $104 K(N \zeta)$ & O51 & $\mathrm{H}$ bond \\
\hline $104 K(N \zeta)$ & O41 & Electrostatic \\
\hline $101 R\left(N \eta_{2}\right)$ & $\mathrm{O} 28$ & $\mathrm{H}$ bond \\
\hline $101 R\left(N \eta_{2}\right)$ & Phenyl ring- $\mathrm{A}$ & Cation- $\pi$ \\
\hline $179 K(N \zeta)$ & $017 ; 014$ & $\mathrm{H}$ bond \\
\hline $103 E\left(O \varepsilon_{1}\right)$ & O51 & $\mathrm{H}$ bond \\
\hline $52 R(C \delta)$ & C61 & Hydrophobic \\
\hline $52 R(C \delta)$ & $\mathrm{C} 62$ & Hydrophobic \\
\hline $76 \mathrm{E}\left(\mathrm{O} \varepsilon_{1}\right)$ & N74 & Electrostatic \\
\hline $76 \mathrm{E}\left(\mathrm{O} \varepsilon_{2}\right)$ & 050 & $\mathrm{H}$ bond \\
\hline $78 \mathrm{~S}(\mathrm{O} \gamma)$ & 050 & $\mathrm{H}$ bond \\
\hline $128 \mathrm{~S}(\mathrm{O} \gamma)$ & $\mathrm{O} 7$ & $\mathrm{H}$ bond \\
\hline $152 \mathrm{Q}\left(\mathrm{N} \varepsilon \mathrm{H}_{1}\right)$ & S9 & $\mathrm{H}$ bond \\
\hline $153 \mathrm{D}\left(\mathrm{O} \delta_{2}\right)$ & 08 & $\mathrm{H}$ bond \\
\hline $153 \mathrm{D}\left(\mathrm{O} \delta_{2}\right)$ & 017 & $\mathrm{H}$ bond \\
\hline $153 \mathrm{D}\left(\mathrm{O} \delta_{1}\right)$ & N15 & $\mathrm{H}$ bond \\
\hline
\end{tabular}

${ }^{\infty} \mathrm{BSR}$ are italicised. ${ }^{\mathrm{t}}$ Refer to Figure S4B for structural details.

doi:10.1371/journal.pone.0064475.t007 out to understand whether single residue or synergistic cooperativity of multiple residues are necessary to generate the binding specificity that operates in gonadotropins and their receptors. It is highly likely that the crucial function of binding specificity of gonadotropins has been entrusted to multiple residues.

\section{Peptidomimetics designed based on BSRs}

Pharmacophore-shape similarity based approach for identification of suitable FSH peptidomimetics. A pharmacophore model was generated based on the identified BSRs of hFSH $\beta$ using pepMMsMIMIC web server. This model was used to retrieve peptidomimetic candidates from 3.9 million commercially available chemical structures present in $\mathrm{MMsINC}^{\circledR}$ database (see Methods). From the retrieved 600 molecules, the peptidomimetic, MMs02514408 was selected since it displayed a) maximum pharmacophoric similarity to the BSRs; b) best docked solution with hFSHR and c) maximum interactions with BSRs of hFSHR (Figure S3). Comparison of the best docked solutions obtained using GOLD and Glide indicated opposite binding modes of MMs02514408 with hFSHR (Figure 3A). Examination of the overlaid docked poses revealed that all the BSRs (except 179K) interacted with MMs02514408 (See Table S2).

Generating novel molecules using fragment-based approach. Fragment-based approach was adopted to generate novel molecules with higher binding affinity to hFSHR. MMs02514408 was fragmented and docked to hFSHR using Fragmentor script and Glide of Schrödinger. The docked fragments were subjected to the popular BREED algorithm to generate 105 novel molecules (see Methods). The best peptidomimetic (FSHP_FB) was selected based on the analysis of the docked poses of these molecules with hFSHR. FSHP_FB was selected since it displayed high binding affinity for hFSHR and interacted with all BSRs including 179K (See Table S3).

Interestingly, unlike MMs02514408, both GOLD and Glide docking algorithms predicted a similar binding orientation of FSHP_FB with hFSHR (Figure 3B). The differential positioning of the aromatic ring of MMs02514408, as observed in the superpositioned GOLD and Glide docked poses, reveal the presence of two hydrophobic ring binding centres on hFSHR. Incidentally, 

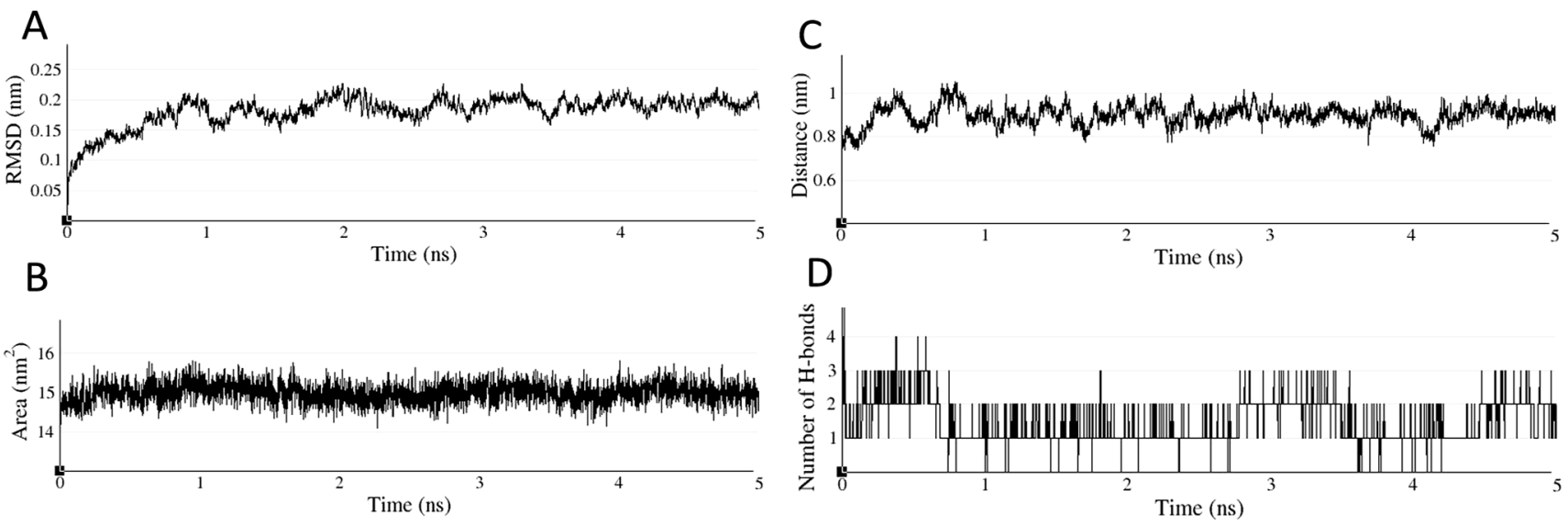

Figure 6. MD trajectory analysis of hFSHR-FSHP complex. (A) Backbone RMSD (B) Total SASA ${ }^{\xi}$ (C) Distance ${ }^{\xi}$ and (D) Number of H-bonds ${ }^{\xi}$. ${ }^{\xi}$ Calculated between the BSRs of FSHR and FSHP.

doi:10.1371/journal.pone.0064475.g006

FSHP_FB has two aromatic rings separated by conformationally constrained spacer (discussed later). Ring A of FSHP_FB forms cation- $\pi$ interaction with $101 \mathrm{R}$ of hFSHR while ring $\mathrm{B}$ is embedded in the hydrophobic pocket of the binding site (Figure 3G). The presence of two phenyl ring binding centres on hFSHR and the increased binding affinity displayed by FSHP_FB as compared to MMs02514408 pinpoints the positive contribution of the two phenyl rings of the ligand for enhanced binding affinity to hFSHR.

Optimisation and development of FSH peptidomimetic (FSHP). FSHP_FB has three stereocentres with R-configuration viz., $11 C \alpha_{1}-1,21 C \alpha_{2}-2$ and $53 C \alpha_{3}-3$. The configuration of the first

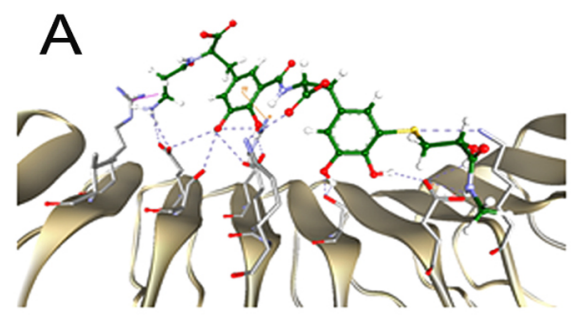

Ons

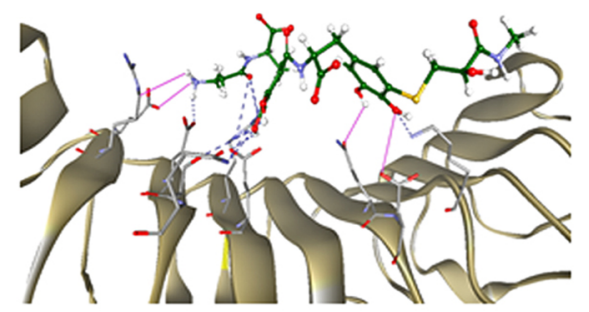

2ns

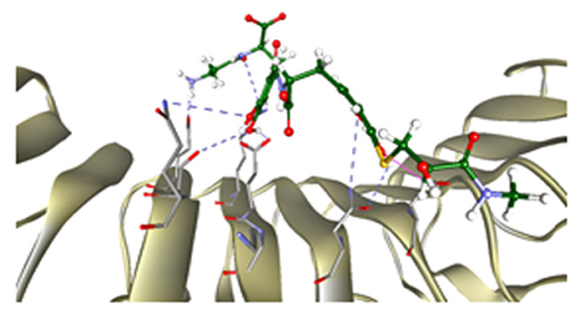

4ns

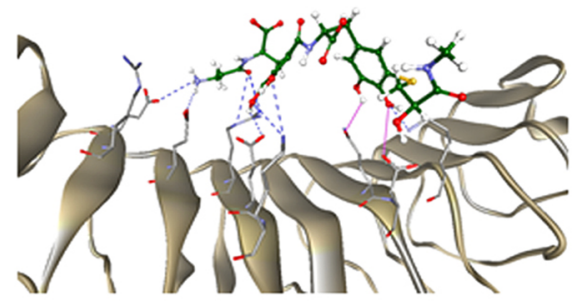

Ins

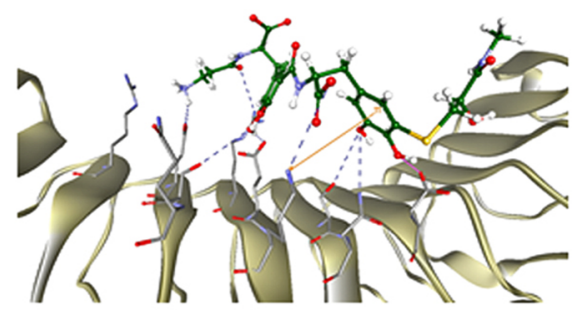

3ns

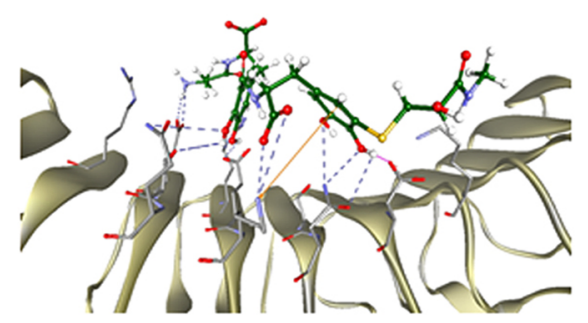

Sns

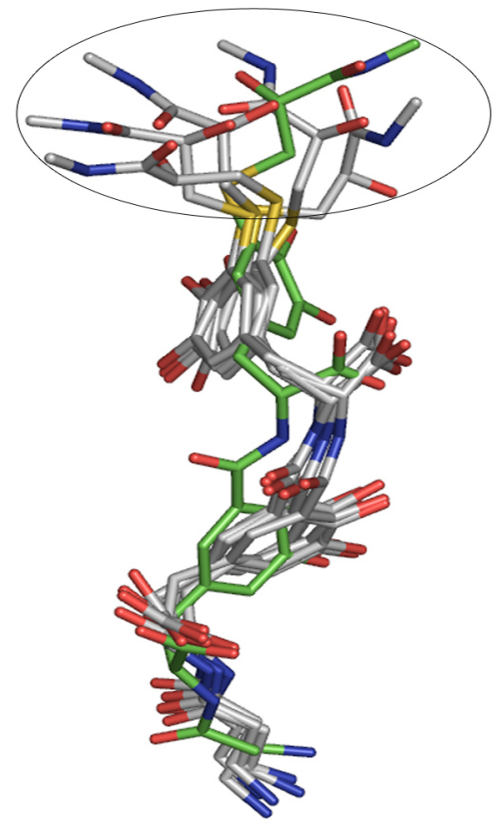

Figure 7. Snapshots of hFSHR-FSHP complex during the course of MD simulation. (A) The binding mode and interactions of FSHP (green ball and sticks) with hFSHR (cartoon) shown at different time intervals. The hydrogen bonds, electrostatic and cation- $\pi$ interactions are shown in blue, pink and orange lines respectively. (B) Molecular overlay of FSHP conformations taken at intervals of 1 ns. The conformation of FSHP taken at 0 ns is shown in green sticks. The region of FSHP that displays flexibility during the simulation is circled.

doi:10.1371/journal.pone.0064475.g007 
A

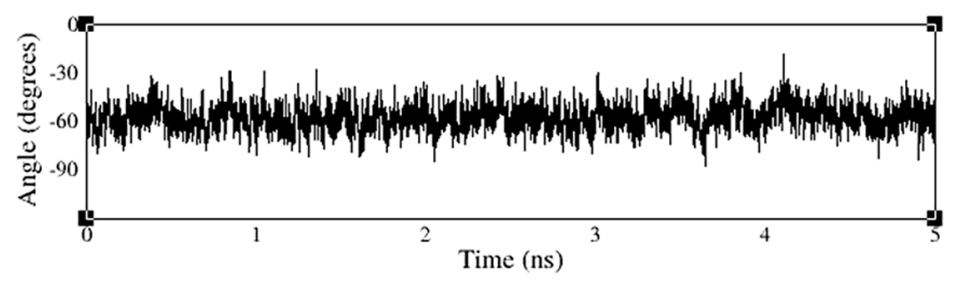

Dihedral Distribution: $57 \mathrm{~N}-53 \mathrm{C} \alpha-52 \mathrm{C} \beta-45 \mathrm{C} \gamma$ average angle: $-57.0772^{\circ}$

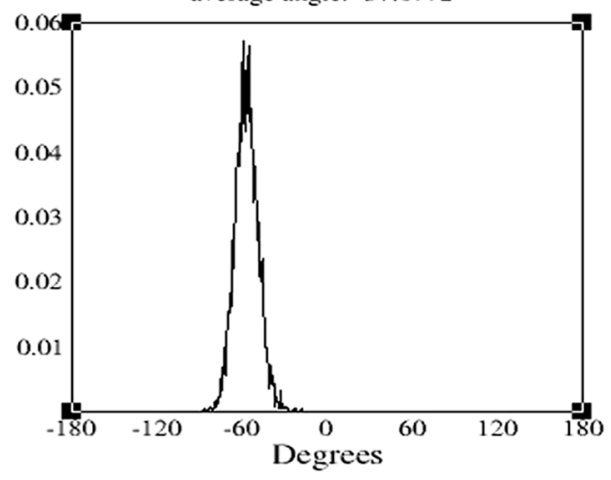

Dihedral Distribution: $25 \mathrm{~N}-21 \mathrm{C} \alpha-20 \mathrm{C} \beta-2 \mathrm{C} \gamma$ average angle: $-57.4972^{\circ}$

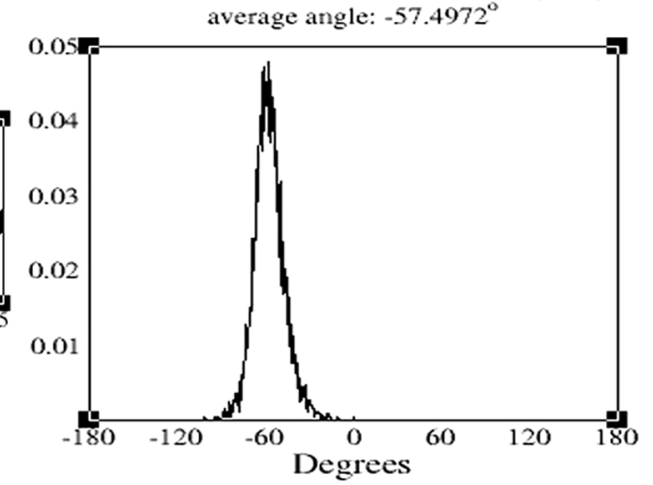

Figure 8. Analysis of two dihedral angles of FSHP during MD simulation. The average dihedral angles and probability distributions plotted for (A) $57 \mathrm{~N}-53 \mathrm{C} \alpha-52 \mathrm{C} \beta-45 \mathrm{C} \gamma$ (B) $25 \mathrm{~N}-21 \mathrm{C} \alpha-20 \mathrm{C} \beta-2 \mathrm{C} \gamma$. doi:10.1371/journal.pone.0064475.g008

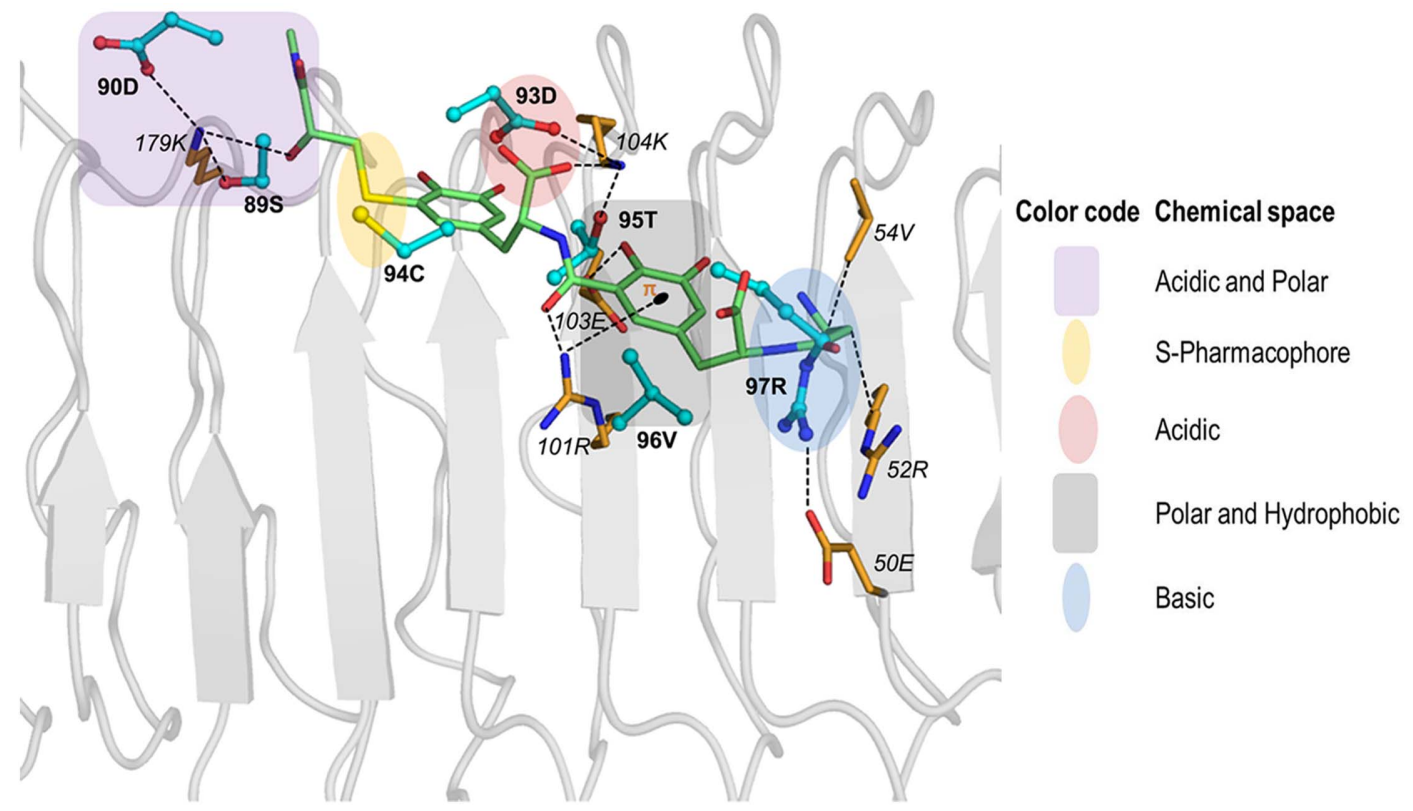

Figure 9. Pharmacophoric similarity of FSHP and hFSH $\beta$. The chemical space shared by hFSH $\beta$ (cyan sticks) and FSHP (green sticks) is colorcoded as explained in the side panel. The interactions with the BSRs (orange sticks) of hFSHR (grey cartoon) are shown in black dotted lines. FSHP shares similar pharmacophoric features and molecular interactions with BSRs of hFSHR as hFSH $\beta$. doi:10.1371/journal.pone.0064475.g009 

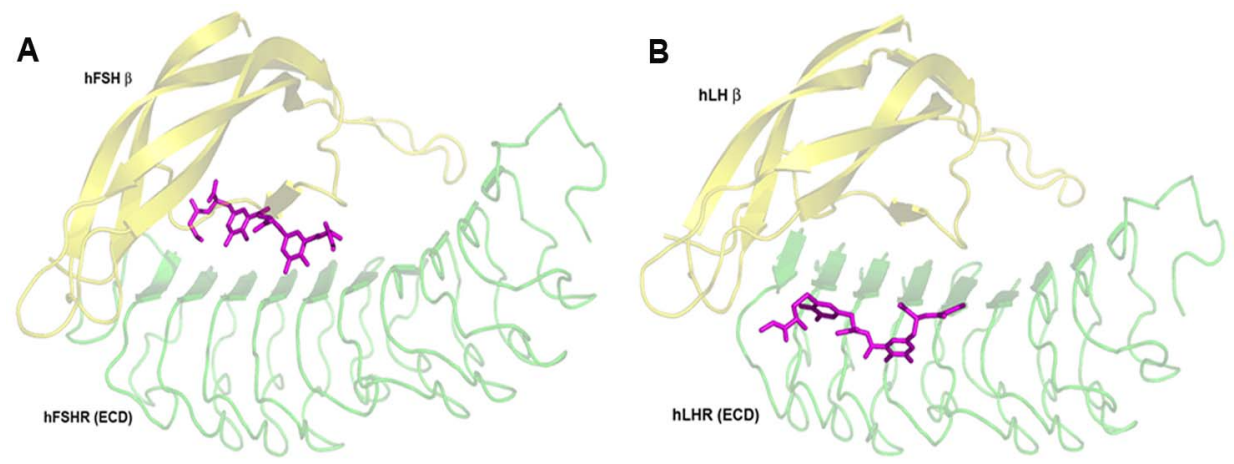

Figure 10. Differential binding modes of FSHP to hFSHR and hLHR. Structures of gonadotropins (yellow cartoon) complexed with their cognate receptors (green cartoon) are super positioned with docked FSHP (pink sticks)-receptor complexes. (A) FSHP binds to hFSHR in a similar mode as that of hFSH $\beta$ (B) FSHP binds to hLHR at a site distant from the hLH $\beta$ binding site.

doi:10.1371/journal.pone.0064475.g010

stereo centre is similar to L-cysteine while the latter two are similar to D-phenylalanine. The solvent accessible formamide (at $\left.11 C \alpha_{1}\right)$ and carboxyl (at $53 C \alpha_{3}$ ) groups were modified to reduce the conformational flexibility and molecular weight. The carboxyl group at $21 \mathrm{C} \alpha_{2}$ position was not modified since its anionic oxygen is involved in salt bridge formation with cationic nitrogen of $104 \mathrm{~K}$ of hFSHR. Additionally, the oxygen is also involved in intramolecular H-bonding with hydroxyl group at the ortho-position of phenyl ring A in FSHP_FB. These interactions assist in reducing the conformational flexibility of the phenyl rings of FSHP_FB (Figure 4).

Twelve peptidomimetics were generated by substituting the solvent accessible formamide (at $11 C \alpha_{1}$ ) and carboxyl (at $53 C \alpha_{3}$ ) groups with different functionalities (Figure 5). These peptidomimetics were docked with hFSHR. The peptidomimetic, FSHP displayed maximum number of interactions with BSRs and exhibited favourable GlideScore, Emodel and non-bonded interaction energies, which were specifically calculated between the BSRs and the docked peptidomimetics (Table 6). The hydroxyl group at $11 C \alpha_{1}$ position of FSHP mimics $89 \mathrm{~S}$ of $\mathrm{hFSH} \beta$ and engages in native-like interaction with $179 \mathrm{~K}$ of hFSHR as observed in the docked complex.

The presence of carboxyl (at $53 C \alpha_{3}$ ) group in FSHP constrains the $57 \mathcal{N}-53 C \alpha-52 C \beta-45 C \gamma$ dihedral angle to energetically favourable gauche-(-), $72.5^{\circ}$ conformation (in case of FSHP_FB, the dihedral angle was gauche-(+), $\left.60^{\circ}\right)$. This dihedral angle is critical

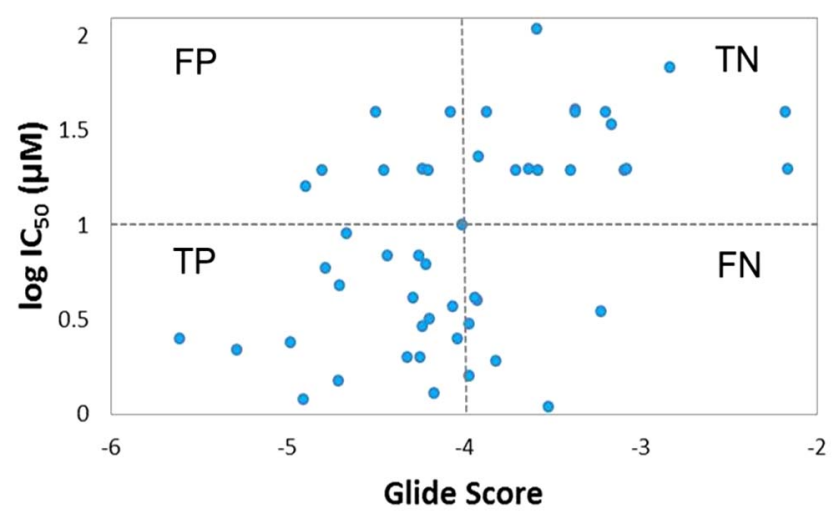

Figure 11. Plot of experimentally determined log IC50 values of $\mathbf{5 0}$ compounds versus their Glide scores.

doi:10.1371/journal.pone.0064475.g011 for placement of the terminal cationic nitrogen (N70) of FSHP in a manner that can mimic the 97R BSR of hFSH $\beta$. This conformation also facilitates cation- $\pi$ interaction between $101 \mathrm{R}$ of hFSHR and phenyl ring A of FSHP (Table 7, Figure S4).

\section{MD simulation studies confirms stability of the hFSHR- FSHP complex}

The stability of the hFSHR-FSHP docked complex was assessed by performing MD simulations for 5 ns. The RMSD of the backbone atoms was calculated with respect to the starting structure as a function of time. The complex achieved stability with an RMSD value around $0.17 \mathrm{~nm}$ within $1 \mathrm{~ns}$ (Figure 6A). The radius of gyration indicated that the structures persisted in the bound state and fluctuated marginally by $0.3 \AA$ relative to the initial structure (Figure S5c). The potential energy plotted as a function of time was found to be stable beyond $0.3 \mathrm{~ns}$ (Figure S5d).

We analysed the trajectory for total solvent accessible surface area (SASA), distance and number of hydrogen bonds between BSRs of hFSHR and FSHP (Figures 6B-D). SASA and the distance between FSHP and the BSRs $(\sim 0.9 \mathrm{~nm})$ did not fluctuate significantly during the course of simulation. We also noticed that FSHP primarily formed 2 hydrogen bonds throughout the simulation with maximum of 4 hydrogen bonds as observed in the docked pose. The above analysis confirms that FSHP stably occupies the binding site of hFSHR throughout the course of simulation.

The average RMS fluctuation was plotted to estimate the extent of residue-wise fluctuations in the hFSHR interface region when bound to FSHP (Figure S5b). During the entire course of simulation, all the BSRs showed minimal fluctuations within the range of $0.05-0.1 \mathrm{~nm}$ (Figure S5a). The low RMS fluctuations indicate that the binding region is rigid and the complex is tightly bound. Snapshots of the intermolecular interactions of hFSHRFSHP taken at $1 \mathrm{~ns}$ intervals of MD simulation are shown in Figure 7A. The overlay of the six representative FSHP conformations reveals that the structure is conformationally rigid except at the 2-hydroxy-N-methyl-propanamido termini (Figure 7B).

To quantify the conformational flexibility of FSHP when bound to hFSHR, we evaluated the distribution of two dihedral angles viz., $57 \mathcal{N}-53 C \alpha-52 C \beta-45 C \gamma$ and $25 \mathcal{N}-21 C \alpha-20 C \beta-2 C \gamma$ which form an important part of the backbone scaffold of FSHP during the course of MD simulation. Dihedral transitions were absent throughout the simulation and the average dihedral angles for $57 \mathcal{N}-53 C \alpha-52 C \beta-45 C \gamma$ and $25 \mathcal{N}-21 C \alpha-20 C \beta-2 C \gamma$ were found to be gauche-(-), $57.07^{\circ}$ and $57.49^{\circ}$ respectively (Figures $8 \mathrm{~A}$ and $\mathrm{B}$ ). 

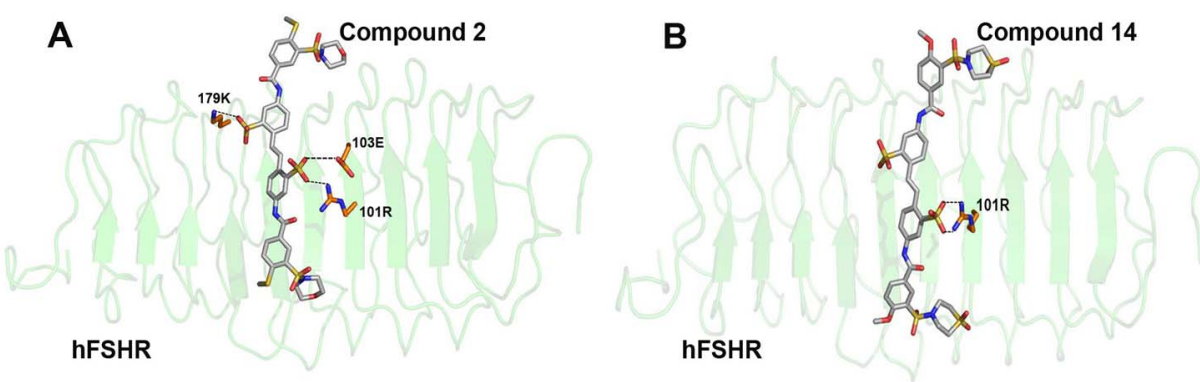

Figure 12. Docked complexes of hFSHR with (A) Compound 2 (B) Compound 14. hFSHR is shown in cartoon representation (green) and the compounds are shown in grey sticks. The compounds display minimal interactions (black dotted lines) with the BSRs (orange sticks). doi:10.1371/journal.pone.0064475.g012

This confirms that FSHP is conformationally rigid and the carboxyl groups at $21 C \alpha_{2}$ and $53 C \alpha_{3}$ positions help in constraining the angles in an energetically favourable conformation.

\section{FSHP has distinct pharmacophoric similarity to hFSH $\beta$}

Examination and comparison of the bound structures of hFSHR-FSH and hFSHR-FSHP indicate that FSHP has distinct pharmacophoric similarity to the binding site of hFSH $\beta$. The docked structures reveal that all the pharmacophoric functionalities of FSHP mimic the physicochemical properties of the BSRs of hFSH $\beta$; such as, i) hydroxyl group at $11 C \alpha_{1}$ position mimics $89 \mathrm{~S}$; ii) pyrocatechol group (ring A) mimics $95 \mathrm{~T}$ and $96 \mathrm{~V}$ and iii) terminal alkyl amino group mimics 97R. FSHP has pharmacophoric groups that also mimic the neighbouring residues of BSRs of $\mathrm{hFSH} \beta$, such as carboxyl group at $21 \mathrm{C} \alpha_{2}$ position and sulfide group of FSHP mimics $93 \mathrm{D}$ and $94 \mathrm{C}$ of $\mathrm{hFSH} \beta$ respectively (Figure 9).

This pharmacophoric similarity explains the high binding affinity of FSHP to hFSHR, as observed from docking and MD studies and also validates its proposed FSH peptidomimetic function.

\section{FSHP specifically mimics the binding mode of $h F S H \beta$ and} not hLH $\beta$

Comparison of the results obtained from docking FSHP to hFSHR and hLHR revealed that while FSHP mimics the binding mode of hFSH $\beta$, it distinctly differs with respect to the binding mode adopted by hLH $\beta$ (Figure 10). FSHP interacts with only one BSR of hLHR (178Y) as compared to five BSRs of hFSHR. This is not surprising as FSHP was designed based on the BSRs of hFSH. These results indicate that FSHP specifically mimics hFSH and not hLH.

Table 8. Chemical names of the designed peptidomimetics.
FSHP has better binding affinity to hFSHR as compared to two known hFSH antagonists

The docking protocol was validated using compounds that were experimentally tested for their potential to bind to the ECD region of hFSHR [62]. 50 such compounds with experimentally determined IC50 values were docked with the ECD region of hFSHR using Glide. Of the 50, 27 compounds had an IC50 value of $<10 \mu \mathrm{M}$ and were considered as actives (True positives) and the remaining 23 compounds were considered as inactives (True negatives). 20 of the 27 actives and 16 of the 23 inactives were predicted correctly as True positives and True negatives respectively. 7 of the actives and 7 of the inactives were wrongly predicted as False negatives and False positives respectively (Figure 11, see Table S4). The sensitivity and specificity of the docking protocol was found to be $\sim 0.7$. Of the 27 actives, three FSH antagonists viz. Compounds 2, 14 and 50 were reported to bind to the ECD region of hFSHR with high affinity and selectivity of which Compounds 2 and 14 exhibited least IC50. Compounds 2 and 14 docked, as expected, to the binding site and exhibited GlideScores of $-4.90 \mathrm{Kcal} / \mathrm{mol}$ (experimental IC50 $1.2 \mu \mathrm{M}$ ) and $-4.32 \mathrm{Kcal} / \mathrm{mol}$ (experimental IC50 $2.0 \mu \mathrm{M}$ ), respectively as compared to a more favourable GlideScore of $7.98 \mathrm{Kcal} / \mathrm{mol}$ for FSHP. The docked poses of both compounds also showed lower number of interactions with BSRs as compared to FSHP (Figure 12).

\section{Conclusions}

The residues crucial for influencing the binding specificity of gonadotropin-receptor complexes have been delineated by sequence and structural analysis. The importance of the identified residues for binding specificity was validated by protein-protein docking with systematic replacement of identified residues and rationalising few examples of cross-reactivity reported in literature for these gonadotropins. The 3D structural information of BSRs of

\begin{tabular}{|c|c|}
\hline Peptidomimetic & Chemical Name \\
\hline MMs02514408 & $\begin{array}{l}\text { (S)-2-amino-5-((R)-3-(5-((R)-2-(3-aminopropanamido)-2-carboxyethyl)-2,3-dihydroxyphenylthio)-1- } \\
\text { (carboxymethylamino)-1-oxopropan-2-ylamino)-5-oxopentanoic acid }\end{array}$ \\
\hline FSHP_FB & $\begin{array}{l}\text { (R)-2"-(5'-((R)-2"-(3-aminopropanamido)-2"-carboxy-ethyl)-3"-(2', 3'-dihydroxybenzamido)-3-(3,4-dihydroxy-5-((R)-2- } \\
\text { formamido-N-methyl-3-thio-propanamido)-phenyl)-propanoic acid }\end{array}$ \\
\hline FSHP & $\begin{array}{l}\text { (R)-2"-(5'-((R)-2"-(3-aminopropanamido)-2"-carboxy-ethyl)-3"-(2', } 3^{\prime} \text {-dihydroxybenzamido)-3-(3,4-dihydroxy-5-((R)-2- } \\
\text { hydroxy-N-methyl-3-thio-propanamido)-phenyl)-propanoic acid }\end{array}$ \\
\hline
\end{tabular}


hFSH $\beta$ was used to design a novel and selective FSH peptidomimetic (FSHP, Table 8) using pharmacophore-shape similarity and fragment-based approach.

While docking results reveal that FSHP can effectively mimic the chemical space of hFSH $\beta$; MD simulations of hFSHR-FSHP complex have shown that FSHP stably interacts with BSRs of hFSHR. Molecular overlay of FSHP conformations, taken at $1 \mathrm{~ns}$ intervals, and the conformational analysis of two important dihedrals of FSHP indicate that FSHP is conformationally rigid except at the 2-hydroxy-N-methyl-propanamido termini connected to phenyl sulfanyl group (Figure 7B). Docking results confirm that FSHP specifically mimics hFSH $\beta$ and not hLH $\beta$. It also has better binding affinity to hFSHR as compared to two known hFSH antagonists. Experimental studies are warranted to confirm the usefulness of FSHP, which is hypothesised to be a good lead candidate for development of fertility regulating agents.

\section{Supporting Information}

Figure S1 Conservation of physicochemical properties of residues involved in binding specificity. $97 \mathrm{R}$ of $\mathrm{hFSH} \beta$ (yellow) is involved in intermolecular interactions with $50 \mathrm{E}$ of hFSHR (green). 50E is stabilized by intra-molecular interaction with second shell residue $52 \mathrm{R}$ of hFSHR. (A) MSA for FSH $\beta$ representing the conserved basic residue $(K, R)$ at position 97 . (B) MSA for LH $\beta$ representing the conservation of $\mathrm{P}$ corresponding to $97^{\text {th }}$ residue of FSH $\beta$.

(TIF)

Figure S2 Validation of ZDOGK algorithm by redocking wild type structures of gonadotropin-receptor complexes. (A) Superposition of X-ray crystal structure of hFSHR (green)FSH (yellow) with the best pose obtained after re-docking (orange) (B) Superposition of modeled structure of hLHR (green)-LH (yellow) with the best pose obtained after re-docking (orange).

(TIF)

Figure S3 (A) Schematic and (B) Chemical structure of MMs02514408, chemical name; (S)-2-amino-5-((R)-3-(5-((R)-2-(3-

\section{References}

1. Audet M, Bouvier M (2012) Restructuring G-protein- coupled receptor activation. Cell 151: 14-23.

2. Gether $\mathrm{U}(2000)$ Uncovering molecular mechanisms involved in activation of $\mathrm{G}$ protein-coupled receptors. Endocrine Reviews 21: 90-113.

3. Weis WI, Kobilka BK (2008) Structural insights into G-protein-coupled receptor activation. Current Opinion in Structural Biology 18: 734-740.

4. Zhang Y, Devries ME, Skolnick J (2006) Structure modeling of all identified G protein-coupled receptors in the human genome. PLoS Computational Biology 2: e13.

5. Shacham S, Topf M, Avisar N, Glaser F, Marantz Y, et al. (2001) Modeling the 3D structure of GPCRs from sequence. Medicinal Research Reviews 21: 472 483.

6. Becker OM, Marantz Y, Shacham S, Inbal B, Heifetz A, et al. (2004) G proteincoupled receptors: In silico drug discovery in 3D. Proceedings of the National Academy of Sciences of the United States of America 101: 11304-11309.

7. Wise A, Gearing K, Rees S (2002) Target validation of G-protein coupled receptors. Drug Discovery Today 7: 235-246.

8. Nambi P, Aiyar N (2003) G protein-coupled receptors in drug discovery. Assay and Drug Development Technologies 1: 305-310.

9. Fox KM, Dias JA, Van Roey P (2001) Three-dimensional structure of human follicle-stimulating hormone. Molecular Endocrinology 15: 378-389.

10. Pierce JG, Parsons TF (1981) Glycoprotein hormones: structure and function. Annual Review of Biochemistry 50: 465-495.

11. Kumar TR (2005) What have we learned about gonadotropin function from gonadotropin subunit and receptor knockout mice? Reproduction 130: 293-302.

12. Moyle WR, Lin W, Myers RV, Cao D, Kerrigan JE, et al. (2005) Models of glycoprotein hormone receptor interaction. Endocrine 26: 189-205.

13. Braun T, Schofield PR, Sprengel R (1991) Amino-terminal leucine-rich repeats in gonadotropin receptors determine hormone selectivity. The EMBO Journal 10: $1885-1890$. aminopropanamido)-2-carboxyethyl)-2,3-dihydroxyphenylthio)-1(carboxymethylamino)-1-oxopropan-2-ylamino)-5-oxopentanoic acid.

(TIF)

Figure S4 Schematic representations of (A) FSHP_FB and (B) FSHP.

(TIF)

Figure S5 Analysis of MD trajectory. (a) Histogram showing the RMSF values for BSRs. (b) RMSF plot showing residue-wise fluctuations of hFSHR (c) Radius of gyration (d) Potential energy.

(TIF)

Table S1 SAVES results for structural validation of hLHR-LH model.

(DOC)

Table S2 Interactions between MMs02514408 and hFSHR in the docked complexes.

(DOG)

Table S3 Interactions between FSHP_FB and FSHR in the docked complexes.

(DOC)

Table S4 Compounds with their experimental IC50 values and Glide Scores on binding with hFSHR.

(DOC)

\section{Acknowledgments}

The authors are grateful to Dr Smita D. Mahale for all the help and support. We thank Padma Nanaware and Swapnil Patil for help in data collection and analysis.

\section{Author Contributions}

Conceived and designed the experiments: AS SN SIT. Performed the experiments: AS SN. Analyzed the data: AS SN. Wrote the paper: AS SN SIT. Principal Investigator of the DBT-funded project: SIT.
14. Vischer HF, Granneman JCM, Noordam MJ, Mosselman S, Bogerd J (2003) Ligand selectivity of gonadotropin receptors. Role of the beta-strands of extracellular leucine-rich repeats 3 and 6 of the human luteinizing hormone receptor. The Journal of Biological Chemistry 278: 15505-15513.

15. Vischer HF, Granneman JCM, Bogerd J (2003) Opposite contribution of two ligand-selective determinants in the N-terminal hormone-binding exodomain of human gonadotropin receptors. Molecular Endocrinology 17: 1972-1981.

16. Vischer HF, Granneman JCM, Bogerd J (2006) Identification of folliclestimulating hormone-selective beta-strands in the N-terminal hormone-binding exodomain of human gonadotropin receptors. Molecular Endocrinology 20: 1880-1893.

17. Campbell RK, Dean-Emig DM, Moyle WR (1991) Conversion of human choriogonadotropin into a follitropin by protein engineering. Proceedings of the National Academy of Sciences of the United States of America 88: 760-764.

18. Dias J, Zhang Y, Liu X (1994) Receptor binding and functional properties of chimeric human follitropin prepared by an exchange between a small hydrophilic intercysteine loop of human follitropin and human lutropin. The Journal of Biological Chemistry 269: 25289-25294

19. Hruby VJ, Cai M (2013) Design of peptide and peptidomimetic ligands with novel pharmacological activity profiles. Annual Review of Pharmacology and Toxicology 53: 557-580.

20. Arkin MR, Wells JA (2004) Small-molecule inhibitors of protein-protein interactions: progressing towards the dream. Nature Reviews Drug Discovery 3: $301-317$

21. González-Ruiz D, Gohlke H (2006) Targeting protein-protein interactions with small molecules: challenges and perspectives for computational binding epitope detection and ligand finding. Current Medicinal Chemistry 13: 2607-2625.

22. Hruby VJ, Balse PM (2000) Conformational and topographical considerations in designing agonist peptidomimetics from peptide leads. Current Medicinal Chemistry 7: 945-970. 
23. Taylor M, Moore S, Mayes J, Parkin E, Beeg M, et al. (2010) Development of a proteolytically stable retro-inverso peptide inhibitor of beta-amyloid oligomerization as a potential novel treatment for Alzheimer's disease. Biochemistry 49: 3261-3272.

24. Floris M, Moro S (2012) Mimicking Peptides... In Silico. Molecular Informatics 31: $12-20$

25. Bhagwanth S, Mishra RK, Johnson RL (2013) Development of peptidomimetic ligands of Pro-Leu-Gly-NH(2) as allosteric modulators of the dopamine $\mathrm{D}(2)$ receptor. Beilstein Journal of Organic Chemistry 9: 204-214.

26. Bruns C, Lewis I, Briner U, Meno-Tetang G, Weckbecker G (2002) SOM230: a novel somatostatin peptidomimetic with broad somatotropin release inhibiting factor (SRIF) receptor binding and a unique antisecretory profile. European Journal of Endocrinology/European Federation of Endocrine Societies 146: 707-716.

27. Ballet S, Salvadori S, Trapella C, Bryant SD, Jinsmaa Y, et al. (2006) New 2', 6'dimethyl-L-tyrosine (Dmt) opioid peptidomimetics based on the Aba-Gly scaffold. Development of unique mu-opioid receptor ligands. Journal of Medicinal Chemistry 49: 3990-3993.

28. Hess S, Linde Y, Ovadia O, Safrai E, Shalev DE, et al. (2008) Backbone cyclic peptidomimetic melanocortin-4 receptor agonist as a novel orally administrated drug lead for treating obesity. Journal of Medicinal Chemistry 51: 1026-1034.

29. Engel JB, Hahne JC, Häusler SFM, Meyer S, Segerer SE, et al. (2012) Peptidomimetic GnRH antagonist AEZS-115 inhibits the growth of ovarian and endometrial cancer cells. Anticancer Research 32: 2063-2068.

30. Straten N van, Timmers M (2009) Non-Peptide Ligands for the Gonadotropin Receptors. Annual Reports in Medicinal Chemistry 44: 171-188.

31. Van Durme J, Horn F, Costagliola S, Vriend G, Vassart G (2006) GRIS: glycoprotein-hormone receptor information system. Molecular Endocrinology 20: 2247-2255.

32. Tina KG, Bhadra R, Srinivasan N (2007) PIC: Protein Interactions Calculator. Nucleic Acids Research 35: W473-476.

33. McDonald IK, Thornton JM (1994) Satisfying hydrogen bonding potential in proteins. Journal of Molecular Biology 238: 777-793.

34. Costantini S, Colonna G, Facchiano AM (2008) ESBRI/: A web server for evaluating salt bridges in proteins. Bioinformation 3: 137-138.

35. Hall TA (1999) BioEdit: a user-friendly biological sequence alignment editor and analysis program for Windows 95/98/NT. Nucleic Acids Symposium Series 41: 95-98.

36. Kabsch W, Sander C (1983) Dictionary of protein secondary structure: pattern recognition of hydrogen-bonded and geometrical features. Biopolymers 22: $2577-2637$.

37. GOLD, version 5.0 User Guide. CGDC Software Ltd.; Cambridge, UK: (2010).

38. Jones G, Willett P, Glen RC, Leach AR, Taylor R (1997) Development and validation of a genetic algorithm for flexible docking. Journal of Molecular Biology 267: 727-748.

39. Floris M, Masciocchi J, Fanton M, Moro S (2011) Swimming into peptidomimetic chemical space using pepMMsMIMIC. Nucleic Acids Research 39: W261-269.

40. Friesner RA, Banks JL, Murphy RB, Halgren TA, Klicic JJ, et al. (2004) Glide: a new approach for rapid, accurate docking and scoring. 1. Method and assessment of docking accuracy. Journal of Medicinal Chemistry 47: 1739-1749.

41. Glide, version 5.7, Schrödinger, LLC, New York, NY, (2011).

42. LigPrep, version 2.5, Schrödinger, LLC, New York, NY, (2011).

43. Maestro, version 9.2, Schrödinger, LLC, New York, NY, (2011).

44. MasciocchiJ, Frau G, Fanton M, Sturlese M, Floris M, et al. (2009) MMsINC: a large-scale chemoinformatics database. Nucleic Acids Research 37: D284-290.
45. Pierce AC, Rao G, Bemis GW (2004) BREED: Generating novel inhibitors through hybridization of known ligands. Application to CDK2, p38, and HIV protease. Journal of Medicinal Chemistry 47: 2768-2775.

46. Loving K, Salam NK, Sherman W (2009) Energetic analysis of fragment docking and application to structure-based pharmacophore hypothesis generation. Journal of Computer-Aided Molecular Design 23: 541-554. doi:10.1007/ s10822-009-9268-1.

47. ChemOffice 2004; CambridgeSoft, 100 CambridgePark Dr. Cambridge, MA 02140 USA

48. Hess B, Kutzner G, Van Der Spoel D, Lindahl E (2008) GROMACS 4: Algorithms for Highly Efficient, Load-Balanced, and Scalable Molecular Simulation. Journal of Chemical Theory and Computation 4: 435-447.

49. Bjelkmar P, Larsson P, Cuendet MA, Hess B, Lindahl E (2010) Implementation of the CHARMM Force Field in GROMACS: Analysis of Protein Stability Effects from Correction Maps, Virtual Interaction Sites, and Water Models. Journal of Chemical Theory and Computation 6: 459-466.

50. Zoete V, Cuendet MA, Grosdidier A, Michielin O (2011) SwissParam: a fast force field generation tool for small organic molecules. Journal of Computational Chemistry 32: 2359-2368.

51. Darden T, York D, Pedersen L (1993) Particle mesh Ewald: An N-log(N) method for Ewald sums in large systems. The Journal of Chemical Physics 98: 10089 10092.

52. Berendsen HJC, Postma JPM, van Gunsteren WF HJ (1981) Inter-molecular forces. In Interaction models for water in relation to protein hydration. Pullman B, editor D Reidel Publishing Company pp.331-342.

53. Bussi G, Donadio D, Parrinello M (2007) Canonical sampling through velocity rescaling. The Journal of Chemical Physics 126: 014101.

54. Nosé S, Klein ML (1983) Constant pressure molecular dynamics for molecular systems. Molecular Physics 50: 1055-1076.

55. Jiang X, Liu H, Chen X, Chen PH, Fischer D, et al. (2012) Structure of folliclestimulating hormone in complex with the entire ectodomain of its receptor. Proceedings of the National Academy of Sciences of the United States of America 109: 12491-12496.

56. Fan QR, Hendrickson W a (2005) Structure of human follicle-stimulating hormone in complex with its receptor. Nature 433: 269-277.

57. Moyle WR, Xing Y, Lin W, Cao D, Myers RV, et al. (2004) Model of glycoprotein hormone receptor ligand binding and signaling. The Journal of Biological Chemistry 279: 44442-44459.

58. Moyle WR, Campbell RK, Myers RV, Bernard MP, Han Y, et al. (1994) Coevolution of ligand-receptor pairs. Nature 368: 251-255.

59. Chopineau M, Martinat N, Troispoux C, Marichatou H, Combarnous Y, et al. (1997) Expression of horse and donkey LH in COS-7 cells: evidence for low FSH activity in donkey LH compared with horse LH. The Journal of Endocrinology 152: $371-377$

60. Noce T, Ando H, Ueda T, Kubokawa K, Higashinakagawa T, et al. (1989) Molecular cloning and nucleotide sequence analysis of the putative cDNA for the precursor molecule of the chicken LH-beta subunit. Journal of Molecular Endocrinology 3: 129-137.

61. Aizen J, Kowalsman N, Kobayashi M, Hollander L, Sohn YC, et al. (2012) Experimental and computational study of inter- and intra- species specificity of gonadotropins for various gonadotropin receptors. Molecular and Cellular Endocrinology 364: 89-100.

62. Wrobel J, Green D, Jetter J, Kao W, Rogers J, et al. (2002) Synthesis of (bis)sulfonic acid, (bis)benzamides as follicle-stimulating hormone (FSH) antagonists. Bioorganic \& Medicinal Chemistry 10: 639-656. 